\title{
Elementos nucleadores da paisagem influenciam a dispersão zoocórica em áreas campestres alto-montanas
}

\author{
Landscape nucleating elements influence the zoochorous dispersion \\ in upper-montane grasslands
}

\author{
Juliana Pizutti Dallabrida ${ }^{1,2}$, Ana Carolina da Silva ${ }^{1}$, Pedro Higuchi ${ }^{1}$, Karine Souza ${ }^{1}$, Rodineli Loebens ${ }^{1}$, \\ Luiz Carlos Rodrigues Júnior ${ }^{1}$, Vanessa Fátima Soboleski ${ }^{1}$, Janaina Gabriela Larsen ${ }^{1}$, \\ Felipe Fornara Walter ${ }^{1} \&$ Ricardo de Vargas Kilca ${ }^{1}$
}

\begin{abstract}
Resumo
Objetivou-se determinar a influência dos elementos nucleadores sobre a regeneração natural de espécies lenhosas, em três áreas de campo, adjacentes a fragmentos de Floresta Ombrófila Mista Alto-Montana. Foram instaladas três transecções, uma por área, perpendiculares à borda dos fragmentos, com sua extensão cobrindo a área de campo. Amostraram-se todos os indivíduos regenerantes arbustivo-arbóreos com $20 \mathrm{~cm}$ ou mais de altura e DAP $<5 \mathrm{~cm}$, que foram classificados em zoocóricos, anemocóricos ou autocóricos. Os indivíduos adultos (DAP $\geq 5 \mathrm{~cm}$ ), troncos caídos, xaxins mortos e rochas foram testados como elementos nucleadores. Obtiveram-se as coordenadas espaciais x e y dos regenerantes e elementos nucleadores, e as propriedades físico-químicas, o grau de compactação dos solos e a topografia. Determinaram-se as relações de dependência espacial entre os indivíduos das guildas de dispersão e os elementos nucleadores por meio da função O-rings. As relações espaciais entre os indivíduos das diferentes síndromes de dispersão e os elementos nucleadores foram distintas entre as áreas. Somente os indivíduos de espécies zoocóricas se associaram aos elementos nucleadores, sendo os mais efetivos os xaxins mortos, troncos e rochas. As condições ambientais distintas entre as áreas imprimiram diferentes padrões de regeneração e relação com os elementos nucleadores. Palavras-chave: distribuição espacial, expansão florestal, síndromes de dispersão de propágulos.
\end{abstract}

\begin{abstract}
We aimed to determine the influence of nucleating elements on woody species regeneration, in three grassland areas, adjacent to fragments of Upper-montane Araucaria Forest. For this, three transects were allocated, one in each area, perpendicular to the fragments edges, with its extension covering the grassland area. All regenerative individuals of shrub-tree species greater than or equal to $20 \mathrm{~cm}$ height up to $\mathrm{dbh}<5 \mathrm{~cm}$ were sampled and classified as zoochoric, anemochoric and autochoric. Adults individuals ( $\mathrm{dbh} \geq 5 \mathrm{~cm})$, fallen stems, dead arborescent ferns, rocks were tested as nucleating elements. The spatial coordinates $(\mathrm{x}, \mathrm{y})$ of regenerative individuals and nucleating elements, soil physicochemical properties, soil compaction degree, and topography were obtained. The spatial dependencies between individuals of different dispersal guilds and nucleating elements were determined by O-rings statistics. The spatial relationships between individuals of distinct dispersal syndromes and nucleating elements differed among areas. Only individuals of zoochoric species presented association with nucleating elements, being the dead arborescent ferns, fallen stems and rocks the most effective elements. The different environmental conditions among areas resulted in distinct regeneration patterns and spatial relationship with nucleating elements.
\end{abstract}

Key words: spatial distribution, forest expansion, propagules dispersal syndromes.

\footnotetext{
${ }^{1}$ Universidade do Estado de Santa Catarina, Depto. Engenharia Florestal, Av. Luiz de Camões 2090, 88520-000, Lages, SC, Brasil.

${ }^{2}$ Autor para correspondência: juli_ajuri@hotmail.com
} 


\section{Introdução}

A Floresta Ombrófila Mista (FOM) ocorre, em muitos locais do sul do Brasil, em mosaicos vegetacionais com áreas de campo natural ou antrópico. No entanto, muitas dessas áreas de campo estão em condições climáticas favoráveis ao desenvolvimento de florestas (Pillar 2003; Müller et al. 2012). Assim, observa-se a tendência da Floresta Ombrófila Mista se expandir sobre o campo (Klein 1960; Behling et al. 2004), por meio do avanço da borda de fragmentos florestais e/ou aos saltos, por meio do estabelecimento de árvores isoladas que funcionam como elementos nucleadores na matriz campestre (Klein 1960). Alguns exemplos de expansão da Floresta Ombrófila Mista associado a elementos nucleadores foram relatados por Oliveira \& Pillar (2004), que observaram maior avanço da floresta em áreas onde o campo apresentava fisionomia arbustiva alta, com os arbustos funcionando como nucleadores. Além disso, Duarte et al. (2006) constataram que indivíduos de Araucaria angustifolia (Bertol.) Kuntze estabelecidos no campo facilitaram o recrutamento de outras espécies florestais, funcionando, também, como elementos nucleadores. Já Carlucci et al. (2011) destacaram a importância de pequenos afloramentos rochosos no campo relevantes para a regeneração de espécies florestais. Dessa forma, é consenso que elementos nucleadores têm atuado como facilitadores da regeneração natural em áreas de campo.

Para Yarranton \& Morrison (1974), o processo de nucleação prevê que a partir de núcleos observados na paisagem, os espaços entre eles podem ser, progressivamente, ocupados por uma diversidade compatível com o conjunto de aptidões bióticas e abióticas. Em estudo mais recente, Bechara (2006) afirma que elementos nucleadores constituem micro habitats que atraem várias formas de vida, funcionando como "gatilhos ecológicos", de forma a catalisar o processo sucessional. Segundo Duarte et al. (2006), estes elementos podem servir como poleiros ou abrigo para a fauna dispersora. Neste sentido, identificar os elementos nucleadores e sua relação com a regeneração natural é importante, pois este conhecimento pode contribuir para a definição de estratégias de restauração ecológica, principalmente em áreas de campos formados a partir de atividades antrópicas.

No entanto, pouco se conhece sobre a relação espacial entre os indivíduos regenerantes arbustivoarbóreos com os elementos nucleadores, em diferentes condições ambientais. Para relacionar estes elementos, pode ser utilizada a função O-ring (Wiegand et al. 1999), ferramenta capaz de detectar agregação ou dispersão a uma dada distância entre as variáveis envolvidas (Wiegand \& Moloney 2004). Assim, o presente estudo teve como objetivo determinar a influência dos elementos nucleadores da paisagem sobre a regeneração de indivíduos arbustivo-arbóreos em três áreas de campo, com diferentes condições ambientais, adjacentes a fragmentos de Floresta Ombrófila Mista AltoMontana, identificando os elementos que possuem maior capacidade de formar núcleos. Espera-se que esse estudo possa gerar subsídios para a recuperação de áreas degradadas em condições semelhantes.

\section{Material e Métodos}

$\mathrm{O}$ estudo foi realizado em três áreas de campo em processo inicial de sucessão florestal, adjacentes a fragmentos florestais, localizadas no Parque Nacional de São Joaquim. O Parque, criado em 1961, abrange cinco municípios de Santa Catarina. As áreas estudadas localizam-se no município de Urubici. A região possui clima $\mathrm{Cfb}$, pela classificação de Köppen e, de acordo com a base de dados do Wordclim (Hijmans et al. 2005), para o período de 1950 a 2000 , a precipitação média anual foi de $1.753 \mathrm{~mm}$ e a temperatura média anual foi de $12,7^{\circ} \mathrm{C}$, com as temperaturas baixas do inverno permitindo a ocorrência de geadas e neve. A vegetação natural do Parque é formada por um mosaico de campos e florestas nebulares, que podem ser classificadas como Floresta Ombrófila Mista Alto-Montana pelo IBGE (2012). Assim, as áreas de floresta são descontínuas, formando fragmentos naturais. Além dos fragmentos naturais, há fragmentos antrópicos formados pela exploração madeireira e criação de gado no passado. Os solos predominantes são pouco profundos, classificados, principalmente, como Neossolos Litólicos e Cambissolos (EMBRAPA 2006) e o relevo varia de suavemente ondulado a fortemente ondulado.

Informações referentes aos históricos das áreas foram fornecidas por meio do setor responsável pela administração do Parque, sendo que a primeira área (Área 1) inventariada, localizada a $28^{\circ} 5$ '41,5”S, 49³0'14,71"W, com altitude do ponto central de $1.628 \mathrm{~m}$, tinha como vegetação original a Floresta Ombrófila Mista Alto-Montana, com a presença constante de nebulosidade, o que caracteriza a floresta nebular, e foi desmatada na década de 1960, para ser utilizada como pastagem. A área foi desapropriada em 2007 
e, desde então, está protegida, de forma que se deu início a um processo inicial de sucessão florestal.

A segunda área (Área 2), localizada a 284'46,87'S, 49³0'51,29'W, com altitude do ponto central de $1.356 \mathrm{~m}$, tinha como vegetação original também a Floresta Ombrófila Mista Alto-Montana, porém, sem a presença constante de nuvens, como na Área 1. Esta foi desmatada para exploração madeireira na década de 1980 e, desde então, tem sido manejada com o uso do fogo para a formação de campo e criação de gado. Foi desapropriada em 2008 e, atualmente, encontra-se em estágio inicial de sucessão florestal.

A terceira área (Área 3), localizada a 28 9'49,19'S, 49³6'47,56'W, com altitude de $1.660 \mathrm{~m}$, é relatada como sempre sendo de campo, com a presença de gado desde o século XIX. Foi desapropriada e protegida em 2008 e a regeneração de espécies lenhosas é reduzida, mesmo com a presença de uma floresta nebular, adjacente a esta área.

Em cada uma das áreas, no ano de 2014, realizou-se a caracterização do componente regenerante arbustivo-arbóreo, a caracterização ambiental e a análise de associação espacial entre o componente regenerante e os elementos nucleadores. Para isso, foram instaladas três transecções, uma em cada área, de largura de $20 \mathrm{~m}$ por $100 \mathrm{~m}$ de comprimento, subdivida em sub-parcelas de $10 \times 10 \mathrm{~m}$. Cada transecção contou com 20 sub-parcelas e teve $2.000 \mathrm{~m}^{2}$ de área total. As transecções foram alocadas de forma perpendicular à borda dos fragmentos adjacentes, com sua extensão cobrindo a área de campo. Foram amostrados todos os indivíduos regenerantes pertencentes a espécies arbustivoarbóreas dentro das sub-parcelas com altura igual ou superior a $20 \mathrm{~cm}$ e com DAP (diâmetro a altura do peito, medido a $1,30 \mathrm{~m}$ do solo) inferior $5 \mathrm{~cm}$. Os indivíduos regenerantes foram classificados em síndromes de dispersão, definidas de acordo com a metodologia de van der Pijl (1982), onde: i) indivíduos de espécies de frutos carnosos ou com outros elementos, que evidenciam a dispersão por animais, foram considerados como zoocóricos; ii) indivíduos de espécies com frutos ou sementes aladas ou outros mecanismos para flutuação foram definidos como anemocóricos; e iii) indivíduos de espécies com frutos deiscente, que, ao se abrirem, liberam as sementes por um rápido movimento, foram considerados autocóricos. A determinação dessas síndromes foi realizada a partir de observações em campo dos diásporos e por consultas na literatura científica (Budke et al. 2005; Almeida et al. 2008; Negrini et al. 2012). Os indivíduos arbustivo-arbóreos com 5 $\mathrm{cm}$ ou mais de DAP foram considerados como parte do componente arbóreo adulto e foram testados como elementos nucleadores. Também foram considerados e testados como elementos nucleadores os xaxins mortos caídos sobre o solo, troncos caídos sobre o solo e rochas. Foram obtidas as coordenadas espaciais $\mathrm{x}$ e y de cada indivíduo regenerante amostrado e, também, dos elementos nucleadores, com auxílio de um medidor de distância ultrassônico DME 201 Haglöf, uma bússola e uma trena.

Para cada área, foram determinados as relações de dependência entre os indivíduos de diferentes guildas de dispersão e os elementos nucleadores, por meio da função O-rings (Wiegand et al. 1999), com correção de borda. A vantagem deste método é o fato de o mesmo não ser acumulativo e, desta forma, não ser influenciado pelos indivíduos observados em menores escalas espaciais. Para verificar se o padrão observado diferenciou de um padrão nulo, foram definidos envelopes de completa aleatoriedade, a partir de 999 permutações, o que representa um envelope com um alfa de 0,01. Como a distância da fonte de propágulo e a heterogeneidade ambiental podem influenciar as relações espaciais entre regenerantes e elementos nucleadores, verificou-se a existência de tendência espacial de primeira ordem ao longo da transeção, por meio de testes qui-quadrado e da razão da verossemelhança $(p<0,05)$. No caso de tendência espacial, o que representaria um padrão não-estacionário, a função O-rings foi definida a partir da função de correlação aos pares para processos não-estacionários, usando a função Kcross.inhom, do pacote spatstat (Baddeley \& Turner 2005) da linguagem de programação estatística R (R Development Core Team 2015).

A caracterização ambiental das áreas foi obtida pelas propriedades físico-químicas e grau de compactação dos solos e levantamento topográfico. As propriedades físico-químicas dos solos foram determinadas a partir da coleta de nove amostras no perfil de 0 a $20 \mathrm{~cm}$, em cada uma das $60 \mathrm{sub}$ parcelas. As nove coletas foram homogeneizadas, retirando-se uma amostra composta por sub-parcela. As análises das propriedades físico-químicas foram realizadas na Universidade do Estado de Santa Catarina (UDESC), de acordo com o protocolo da Rede Oficial de Laboratórios de Análise de Solo e Tecidos Vegetais dos estados do Rio Grande do 
Sul e de Santa Catarina (ROLAS), quantificandose: teores de areia, silte e argila, $\mathrm{pH}$ em $\mathrm{H}_{2} \mathrm{O}$, cálcio $(\mathrm{Ca})$, magnésio $(\mathrm{Mg})$, alumínio $(\mathrm{Al})$, sódio $(\mathrm{Na})$, fósforo $(\mathrm{P})$ por Melich, potássio $(\mathrm{K})$, capacidade de troca catiônica (CTC efetiva), soma de bases (V) e matéria orgânica (MO). A compactação do solo foi obtida por meio de testes de resistência à penetração vertical, utilizando um penetrômetro de impacto digital penetrolog PLG1020 Falker, com o cone tipo 2, com dez repetições sistemáticas em cada sub-parcela, na profundidade de 0 a 20 $\mathrm{cm}$. Dessas dez leituras, foi retirada uma média por sub-parcela. Assim, em cada sub-parcela, foi obtido uma leitura de resistência a penetração a $1 \mathrm{~cm}, 2 \mathrm{~cm}$, até $20 \mathrm{~cm}$ de profundidade. Foram consideradas, para a caracterização da resistência a penetração, a média dessas leituras de $1 \mathrm{~cm}$ até 20 $\mathrm{cm}$, a resistência a penetração máxima nesse perfil e a resistência a penetração somente a $20 \mathrm{~cm}$ de profundidade. No levantamento topográfico, três variáveis topográficas foram determinadas em cada sub-parcela: cota, desnível máximo e declividade média. A cota foi calculada pela média das cotas dos quatro vértices da sub-parcela, o desnível máximo correspondeu à maior distância vertical entre os vértices da sub-parcela (Oliveira Filho et al. 1994) e a declividade foi calculada pela média da declividade dos quatro lados da sub-parcela.

As variáveis ambientais foram comparadas entre as áreas. Para isso, foi realizado o teste de Shapiro-Wilk para verificar a normalidade dos dados. Para as variáveis que possuíam distribuição normal, foi realizada a Análise de Variância (ANOVA) para detectar diferenças entre as variáveis e, para as variáveis distintas ( $p \leq$ $0,05)$, foi realizado o teste de Tukey para verificar em qual área ocorreu a diferença. No caso de distribuição não normal, foi utilizado o teste de Kruskal-Wallis para verificar as diferenças entre as variáveis e, para detectar a área diferente, o teste de comparação múltipla. Essas análises foram realizadas na linguagem de programação estatística R (R Development Core Team 2015), com o pacote pgirmess (Giraudoux 2015).

\section{Resultados}

$\mathrm{Na}$ Área 1 foram amostrados 205 indivíduos de espécies anemocóricas e 485 de espécies zoocóricas, não sendo encontrados indivíduos de espécies autocóricas (Fig. 1). Como elementos nucleadores, contabilizaram-se 210 rochas, 171 xaxins mortos, 40 troncos caídos e um indivíduo adulto vivo. Esse último, por ser único, não foi considerado nas análises. Os indivíduos anemocóricos e os elementos nucleadores do tipo rochas apresentaram padrão não estacionário, ou seja, distribuição não uniforme com tendência espacial de primeira ordem. Os indivíduos zoocóricos e os elementos xaxim morto e tronco apresentaram padrão estacionário, ou seja, distribuição uniforme sem tendência espacial de primeira ordem. Os indivíduos anemocóricos da Área 1 não apresentaram associação espacial com os elementos nucleadores (Fig. 2). Para os indivíduos zoocóricos da mesma área, houve associação com
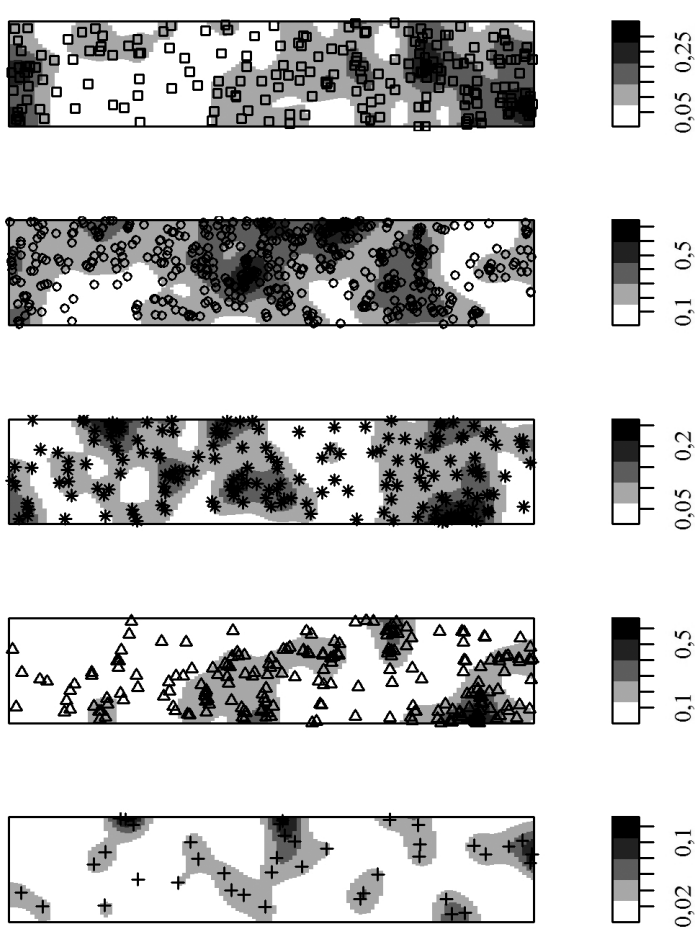

Figura 1 - Distribuição espacial dos indivíduos arbustivo-arbóreos e elementos nucleadores, ao longo da transeção da Área 1, alocada perpendicular a um remanescente florestal de Floresta Ombrófila Mista AltoMontana, situado a esquerda da transecção. Quadrados abertos: anemocóricos; círculos: zoocóricos; asterisco: xaxins mortos; triângulos: rochas; cruz: troncos caídos. Os valores apresentados na escala disposta verticalmente ao lado das ilustrações da transecção referem-se à estimativa de densidade Kernel.

Figure 1 - Spatial distribution of shrub-tree individuals and nucleating elements, along Area 1 transect, allocated perpendicular to an Upper-montane Araucaria Forest fragment, situated at the left of the transect. Open square: anemochorous; circle: zoochorous; asterisk: dead arborescent fern; triangle: rocks; crosse: fallen stems. The scale vertically arranged alongside transect illustrations refers to the estimated kernel density. 
os elementos nucleadores do tipo xaxim morto e tronco, indicando agregação a aproximadamente 1,5 m de distância de cada elemento (Fig. 3).

$\mathrm{Na}$ Área 2 foram encontrados 460 indivíduos de espécies anemocóricas, 131 de espécies zoocóricas e 105 de espécies autocóricas (Fig. 4). Como elementos nucleadores, encontraramse 85 rochas, 33 troncos caídos e sete árvores adultas vivas. Não foram encontrados xaxins mortos. Os indivíduos de todas as guildas de dispersão, bem como os elementos nucleadores dos tipos rocha e árvores adultas, apresentaram padrão não estacionário. Apenas os troncos caídos apresentaram padrão estacionário. Para a Área 2 , também não foram encontradas associações espaciais entre os indivíduos anemocóricos com os elementos nucleadores (Fig. 5), assim como entre os indivíduos autocóricos e esses elementos (Fig. 6). Porém, para os zoocóricos (Fig. 7), houve associação somente com as rochas, a aproximadamente $4 \mathrm{~m}$ de distância desses.
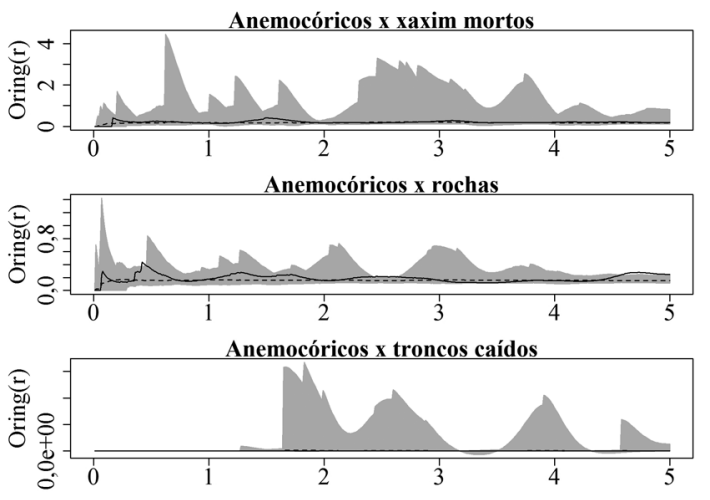

Figura 2 - Relação dos indivíduos anemocóricos com os elementos nucleadores na Área 1, adjacente a um remanescente de Floresta Ombrófila Mista Alto-Montana. Em cinza está o envelope de completa aleatoriedade espacial. A linha contínua representa a relação espacial entre os regenerantes e elementos nucleadores, sendo que a linha acima desse envelope indica agregação entre os elementos, e linha abaixo, segregação entre os elementos. Valores representados pelo eixo $\mathrm{x}$ indicam a distância em metros $(\mathrm{m})$ do elemento nucleador.

Figure 2 - Relation of anemochorous individuals with nucleating elements in Area 1, adjacent to an Upper-montane Araucaria Forest fragment. The gray area is the envelope of complete spatial randomness. The solid line represents the spatial relationship between the regenerating and nucleating elements. The solid line above the envelope indicates aggregation of elements and the solid line below the envelope indicates segregation of elements. $\mathrm{X}$ axis values indicate the distance in meters $(\mathrm{m})$ from nucleating element.
$\mathrm{Na}$ Área 3, que sempre foi uma área de campo, foram amostrados apenas dois indivíduos regenerantes arbustivo-arbóreos de espécies anemocóricas e quatro de espécies zoocóricas (Fig. 8). Não foram encontrados indivíduos de espécies autocóricas. Como elementos nucleadores da paisagem, encontraram-se 42 rochas. Todos os indivíduos e os elementos nucleadores apresentaram padrão não estacionário, o que era esperado devido à baixa densidade. Não houve relação dos regenerantes com os elementos nucleadores (Fig. 9).

Além dos diferentes históricos das áreas estudadas, essas diferiram na maioria das variáveis ambientais mensuradas (Tab. 1). A análise das variáveis edáficas indicou que a Área 3 caracterizase pela textura mais arenosa dos solos (teor de areia $=83,85 \%$ ), com maior concentração de alumínio $\left(\mathrm{Al}=8,23 \mathrm{cmolc} / \mathrm{dm}^{3}\right)$ e maior resistência a penetração (média $=622,90 \mathrm{kPa})$, em comparação com as Áreas 1 e 2, menos arenosas
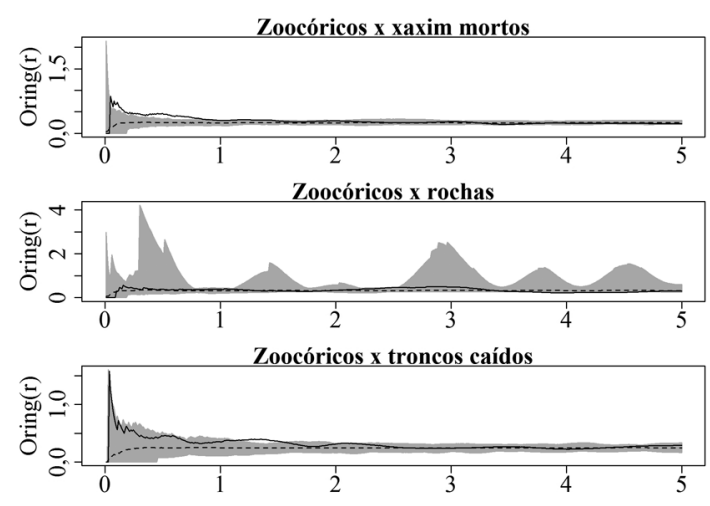

Figura 3 - Relação dos indivíduos zoocóricos com os elementos nucleadores na Área 1, adjacente a um remanescente de Floresta Ombrófila Mista Alto-Montana. Em cinza está o envelope de completa aleatoriedade espacial. A linha contínua representa a relação espacial entre os regenerantes e elementos nucleadores, sendo que a linha acima desse envelope indica agregação entre os elementos, e linha abaixo, segregação entre os elementos. Valores representados pelo eixo $\mathrm{x}$ indicam a distância em metros $(\mathrm{m})$ do elemento nucleador.

Figure 3 - Relation of zoochorous individuals with nucleating elements in Area 1, adjacent to an Upper-montane Araucaria Forest fragment. The gray area is the envelope of complete spatial randomness. The solid line represents the spatial relationship between the regenerating and nucleating elements. The solid line above the envelope indicates aggregation of elements and the solid line below the envelope indicates segregation of elements. $\mathrm{X}$ axis values indicate the distance in meters $(\mathrm{m})$ from nucleating element. 
$(62,95 \%$ e $63,95 \%$, respectivamente $)$, com menores teores de alumínio $(5,02$ e $3,35 \mathrm{cmolc} /$ $\mathrm{dm}^{3}$, respectivamente) e menores resistências a penetração dos solos (médias de 511,30 e 513,30 $\mathrm{kPa}$, respectivamente). No entanto, em relação às outras variáveis químicas dos solos, houve padrões diferenciados. Quanto à acidez, apesar
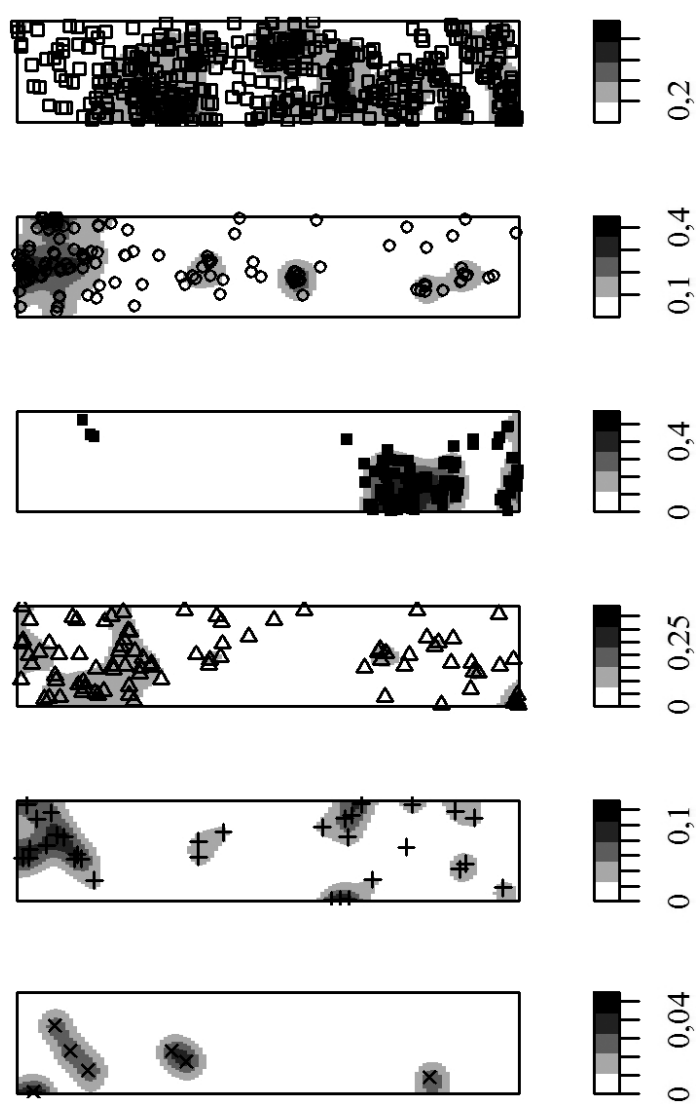

Figura 4 - Distribuição espacial dos indivíduos arbustivoarbóreos e elementos nucleadores, ao longo da transeção da Área 2, alocada perpendicular a um remanescente florestal de Floresta Ombrófila Mista Alto-Montana, situado a esquerda da transecção. Quadrados abertos: anemocóricos; círculos: zoocóricos; quadrados fechados: autocóricos; triângulos: rochas; cruz: troncos caídos; " $\mathrm{x}$ ": árvores adultas. Os valores apresentados na escala disposta verticalmente ao lado das ilustrações da transecção referem-se à estimativa de densidade Kernel.

Figure 4-Spatial distribution of shrub-tree individuals and nucleating elements, along Area 2 transect, allocated perpendicular to an Uppermontane Araucaria Forest fragment, situated at the left of the transect. Open square: anemochorous; circle: zoochorous; closed square: autochorous; triangle: rocks; cross: fallen stems; "x": adult trees. The scale vertically arranged alongside transect illustrations refers to the estimated kernel density. das diferenças significativas entre as áreas, os valores de $\mathrm{pH}$ foram muito semelhantes (entre 4,40 e 4,55), podendo todos os solos serem classificados como ácidos. A Área 3 foi a que obteve os maiores valores de matéria orgânica $(13,80 \%)$, o que contribuiu para o maior valor de CTC nessa área $\left(12,36 \mathrm{cmolc} / \mathrm{dm}^{3}\right)$. No entanto, a Área 3, juntamente com a Área 1, foram as que apresentaram as menores saturações por bases (7,64 e 14,20\%), denotando solos distróficos $(\mathrm{V}<50 \%)$. A Área 2, com somente $25,95 \%$ de saturação de bases, também pode ser classificada como de solos distróficos, porém, com maior fertilidade que a Área 3. Não houve variações topográficas entre as áreas, exceto pela cota, que foi menor na Área 2 (1.338,00 $\mathrm{m}$ de altitude). De forma geral, a análise ambiental demonstrou que a Área 3 foi a mais distinta, apresentando solos mais arenosos, álicos e compactados.
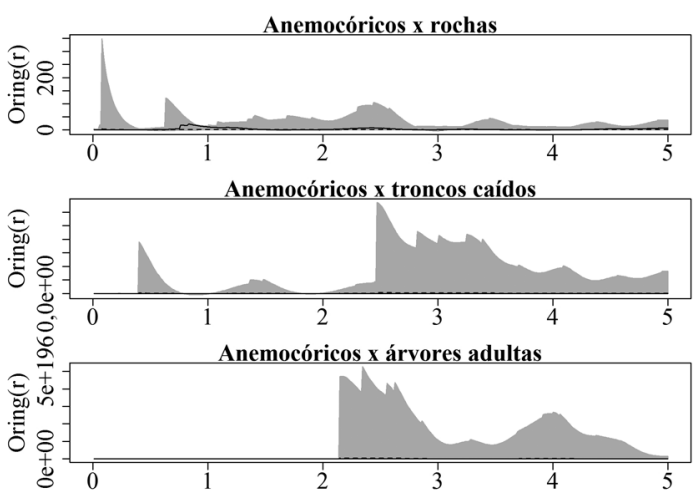

Figura 5 - Relação dos indivíduos anemocóricos com os elementos nucleadores na Área 2, adjacente a um remanescente de Floresta Ombrófila Mista Alto-Montana. Em cinza está o envelope de completa aleatoriedade espacial. A linha contínua representa a relação espacial entre os regenerantes e elementos nucleadores, sendo que a linha acima desse envelope, indica agregação entre os elementos, e linha abaixo, segregação entre os elementos. Valores representados pelo eixo $\mathrm{x}$ indicam a distância em metros $(\mathrm{m})$ do elemento nucleador.

Figure 5 - Relation of anemochorous individuals with nucleating elements in Area 2, adjacent to an Upper-montane Araucaria Forest fragment. The gray area is the envelope of complete spatial randomness. The solid line represents the spatial relationship between the regenerating and nucleating elements. The solid line above the envelope indicates aggregation of elements and the solid line below the envelope indicates segregation of elements. $\mathrm{X}$ axis values indicate the distance in meters $(\mathrm{m})$ from nucleating element. 


\section{Discussão}

O maior número de indivíduos zoocóricos encontrado na Área 1 é um padrão comum no Brasil, onde encontramos a maioria das espécies e, ou, indivíduos zoocóricos em florestas tropicais (e.g., Aquino \& Barbosa 2009; Pereira et al. 2010) ou subtropicais (e.g., Budke et al. 2005; Negrini et al. 2012). Esse padrão indica que a regeneração da área é dependente da fauna. Porém, na Área 2, o padrão de maior parte de anemocóricos, indica o estágio inicial de sucessão dessa área, onde são observados muitos indivíduos de Baccharis, gênero anemocórico com a maioria das espécies pioneiras, além de outras espécies de Asteraceae, também com dispersão pelo vento. No entanto, é importante destacar que a Área 1 também se encontra em estágio inicial de sucessão, porém, apesar do elevado número de indivíduos de Baccharis spp. também nessa área, há elevado número de espécies do gênero
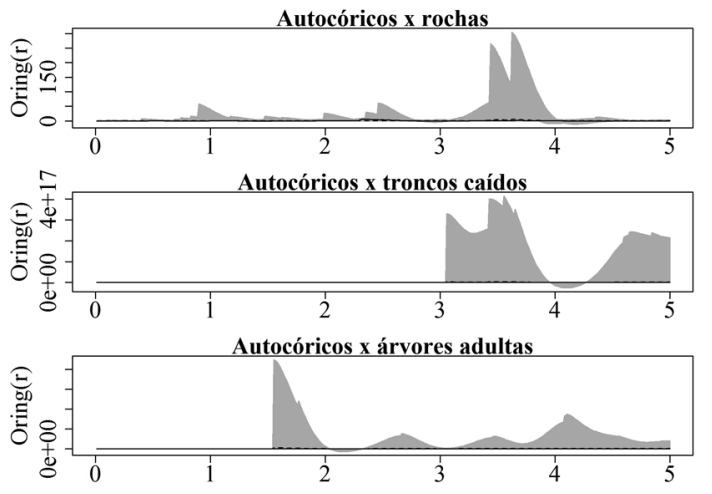

Figura 6 - Relação dos indivíduos autocóricos com os elementos nucleadores na Área 2, adjacente a um remanescente de Floresta Ombrófila Mista AltoMontana. Em cinza está o envelope de completa aleatoriedade espacial. A linha contínua representa a relação espacial entre os regenerantes e elementos nucleadores, sendo que a linha acima desse envelope, indica agregação entre os elementos, e linha abaixo, segregação entre os elementos. Valores representados pelo eixo $\mathrm{x}$ indicam a distância em metros $(\mathrm{m})$ do elemento nucleador.

Figure 6-Relation of autochorous individuals with nucleating elements in Area 2, adjacent to an Upper-montane Araucaria Forest fragment. The gray area is the envelope of complete spatial randomness. The solid line represents the spatial relationship between the regenerating and nucleating elements. The solid line above the envelope indicates aggregation of elements and the solid line below the envelope indicates segregation of elements. $\mathrm{X}$ axis values indicate the distance in meters (m) from nucleating element.
Solanum, com espécies também pioneiras, mas zoocóricas. Na Área 2 também se destacou o elevado número de indivíduos autocóricos, especialmente representados pelo gênero Croton, sendo que na Área 1 nenhum autocórico foi encontrado, o que confirma a elevada distinção entre as áreas.

O padrão espacial de primeira ordem dos diferentes elementos analisados, estacionários ou não, é o resultado dos processos dinâmicos que ocorrem em diferentes escalas no espaço e no tempo (Dale \& Fortin 2014). No caso de rochas, tendências espaciais podem ser reflexo de processos geomorfológicos, como, por exemplo, as diferenças nos processos erosivos ao longo do relevo. No caso das plantas, tendências espaciais podem ser reflexo de processos ecológicos, como a dispersão de propágulos. Do ponto de vista prático, o reconhecimento do tipo de padrão espacial de primeira ordem é importante para a escolha dos
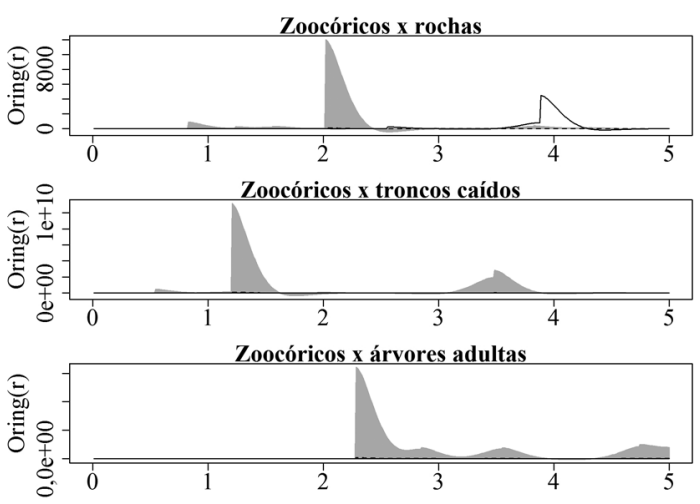

Figura 7 - Relação dos indivíduos zoocóricos com os elementos nucleadores na Área 2, adjacente a um remanescente de Floresta Ombrófila Mista AltoMontana. Em cinza está o envelope de completa aleatoriedade espacial. A linha contínua representa a relação espacial entre os regenerantes e elementos nucleadores, sendo que a linha acima desse envelope, indica agregação entre os elementos, e linha abaixo, segregação entre os elementos. Valores representados pelo eixo $\mathrm{x}$ indicam a distância em metros $(\mathrm{m})$ do elemento nucleador.

Figure 7 - Relation of zoochorous individuals with nucleating elements in Area 2, adjacent to an Upper-montane Araucaria Forest fragment. The gray area is the envelope of complete spatial randomness. The solid line represents the spatial relationship between the regenerating and nucleating elements. The solid line above the envelope indicates aggregation of elements and the solid line below the envelope indicates segregation of elements. $\mathrm{X}$ axis values indicate the distance in meters $(\mathrm{m})$ from nucleating element. 
métodos estatísticos a serem utilizados para a análise da dependência espacial entre os diferentes elementos (Dale \& Fortin 2014). No presente estudo, para a aplicação da função de O-ring, considerouse a natureza estacionária ou não estacionária dos dados, utilizando-se uma função de correlação aos pares específica para cada caso.

A zoocoria foi a única guilda de dispersão cuja presença de elementos nucleadores influenciou o padrão de distribuição espacial dos indivíduos. A Área 3 foi a exceção, visto que neste local foi observada baixa densidade de indivíduos regenerantes para todas as síndromes de dispersão. Esse resultado reforça a ideia de que elementos nucleadores são importantes facilitadores do processo de regeneração natural em áreas abertas (Oliveira \& Pillar 2004), com influência sobre a dispersão realizada por animais (Duarte et al. 2010). Supostamente, animais utilizam os elementos nucleadores como hábitat e/ou, poleiro em todas as áreas, porém, a inexistência de associação na Área 3 pode estar relacionada com fatores limitantes à regeneração natural, como as condições ambientais existentes.
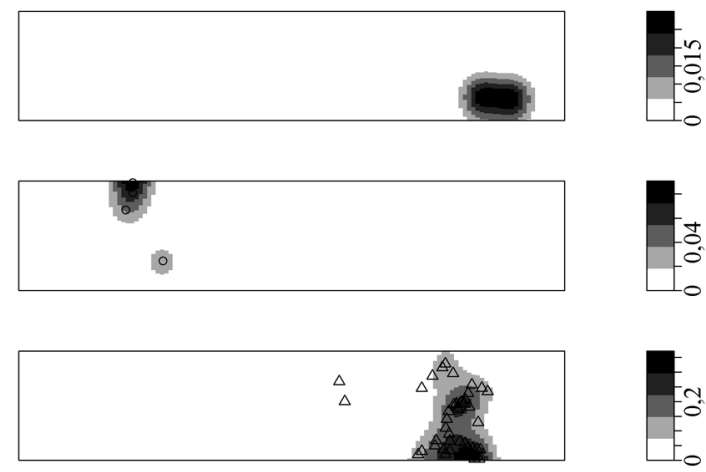

Figura 8 - Distribuição espacial dos indivíduos arbustivo-arbóreos e elementos nucleadores, ao longo da transeção da Área 3, alocada perpendicular a um remanescente florestal de Floresta Ombrófila Mista Alto-Montana, situado a esquerda da transecção. Quadrados abertos: anemocóricos; círculos: zoocóricos; triângulos: rochas. Os valores apresentados na escala disposta verticalmente ao lado das ilustrações da transecção referem-se à estimativa de densidade Kernel. Figure 8 - Spatial distribution of shrub-tree individuals and nucleating elements, along Area 3 transect, allocated perpendicular to an Upper-montane Araucaria Forest fragment, situated at the left of the transect. Open square: anemochorous; circle: zoochorous; triangle: rocks. The scale vertically arranged alongside transect illustrations refers to the estimated kernel density.
Também é interessante observar que as Áreas 1 e 2 diferiram quanto aos elementos nucleadores influentes na distribuição espacial dos indivíduos zoocóricos. No caso da Área 1, infere-se que houve preferência pela fauna, sobretudo, pelos xaxins mortos, provavelmente pelas melhores condições de abrigo fornecidas, e, em segundo lugar, pelos troncos; enquanto que na Área 2, a preferência foi pelas rochas. No entanto, é importante destacar que na Área 2 não havia xaxins mortos e havia somente 33 troncos, em comparação com o maior número de rochas (85). Assim, pode-se inferir que, quando havia xaxins mortos e troncos suficientes, a preferência de utilização pela fauna era desses elementos, porém, na abundância somente de rochas, a fauna utilizou esses elementos, já destacado na literatura como importantes na nucleação. Por exemplo: Pillar (2003) e Müller et al. (2012) destacam que os afloramentos rochosos funcionam como núcleos de expansão florestal
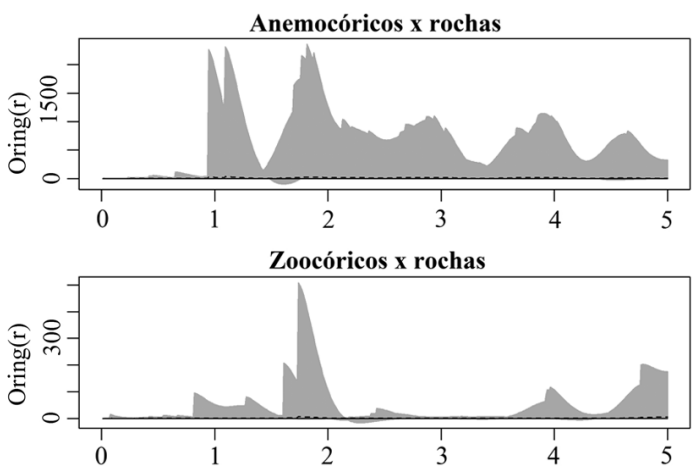

Figura 9 - Relação dos indivíduos anemocóricos e zoocóricos com os elementos nucleadores na Área 3, adjacente a um remanescente de Floresta Ombrófila Mista Alto-Montana. Em cinza está o envelope de completa aleatoriedade espacial. A linha contínua representa a relação espacial entre os regenerantes e elementos nucleadores, sendo que a linha acima desse envelope, indica agregação entre os elementos, e linha abaixo, segregação entre os elementos. Valores representados pelo eixo $\mathrm{x}$ indicam a distância em metros (m) do elemento nucleador.

Figure 9 - Relation of anemochorous and zoochorous individuals with nucleating elements in Area 3, adjacent to an Upper-montane Araucaria Forest fragment. The gray area is the envelope of complete spatial randomness. The solid line represents the spatial relationship between the regenerating and nucleating elements. The solid line above the envelope indicates aggregation of elements and the solid line below the envelope indicates segregation of elements. $\mathrm{X}$ axis values indicate the distance in meters $(\mathrm{m})$ from nucleating element. 
Tabela 1 - Médias das variáveis ambientais obtidas nas Áreas 1, 2 e 3, adjacentes a remanescentes de Floresta Ombrófila Mista Alto-Montana.

Table 1 - Environmental variables means obtained in Areas 1, 2 and 3, adjacent to Upper-montane Araucaria Forest fragments.

\begin{tabular}{|c|c|c|c|c|}
\hline Variáveis & Área 1 & Área 2 & Área 3 & $P$ \\
\hline Areia (\%) & $62,95^{\mathrm{b}}$ & $63,95^{\mathrm{b}}$ & $83,85^{\mathrm{a}}$ & $\mathrm{Kw} 3,11 \mathrm{e}^{-9^{*}}$ \\
\hline Silte (\%) & $24,90^{\mathrm{a}}$ & $25,95^{\mathrm{a}}$ & $12,30^{\mathrm{b}}$ & $\mathrm{Kw} 2,56 \mathrm{e}^{-8^{*}}$ \\
\hline Argila (\%) & $12,10^{\mathrm{a}}$ & $10,25^{\mathrm{a}}$ & $3,90^{\mathrm{b}}$ & $\mathrm{Kw} 2,13 \mathrm{e}^{-9^{*}}$ \\
\hline $\mathrm{pH} \mathrm{em} \mathrm{H}_{2} \mathrm{O}$ & $4,40^{\mathrm{b}}$ & $4,55^{\mathrm{a}}$ & $4,44^{\mathrm{ab}}$ & $\mathrm{Kw} 0,04^{*}$ \\
\hline $\mathrm{Ca}\left(\mathrm{cmolc} / \mathrm{dm}^{3}\right)$ & $3,01^{\mathrm{b}}$ & $4,54^{\mathrm{a}}$ & $3,17^{\mathrm{ab}}$ & $\mathrm{Kw} 4,13 \mathrm{e}^{-5^{*}}$ \\
\hline $\mathrm{Mg}\left(\mathrm{cmolc} / \mathrm{dm}^{3}\right)$ & 0,62 & 0,64 & 0,59 & Av 0,55 \\
\hline $\mathrm{Al}\left(\mathrm{cmolc} / \mathrm{dm}^{3}\right)$ & $5,02^{\mathrm{b}}$ & $3,35^{\mathrm{b}}$ & $8,23^{\mathrm{a}}$ & $\mathrm{Kw} 4,05 \mathrm{e}^{-9^{*}}$ \\
\hline $\mathrm{Na}\left(\mathrm{mg} / \mathrm{dm}^{3}\right)$ & 29,10 & 36,70 & 35,60 & Av 0,12 \\
\hline $\mathrm{P}\left(\mathrm{mg} / \mathrm{dm}^{3}\right)$ & 8,49 & 11,18 & 11,91 & Kw 0,11 \\
\hline $\mathrm{K}\left(\mathrm{mg} / \mathrm{dm}^{3}\right)$ & $87,70^{\mathrm{b}}$ & $84,90^{\mathrm{b}}$ & $147,20^{\mathrm{a}}$ & $\mathrm{Kw} 8,98 \mathrm{e}^{-8^{*}}$ \\
\hline CTC efetiva (cmolc/dm ${ }^{3}$ ) & $8,87^{\mathrm{b}}$ & $8,74^{\mathrm{b}}$ & $12,36^{\mathrm{a}}$ & Kw $1,40 \mathrm{e}^{-7^{*}}$ \\
\hline $\mathrm{V}(\%)$ & $14,20^{\mathrm{ab}}$ & $25,95^{\mathrm{a}}$ & $7,64^{\mathrm{b}}$ & $\mathrm{Kw} 2,76 \mathrm{e}^{-10^{*}}$ \\
\hline MO (\%) & $5,73^{\mathrm{b}}$ & $4,42^{\mathrm{b}}$ & $13,80^{\mathrm{a}}$ & $\mathrm{Kw} 1,22 \mathrm{e}^{-9^{*}}$ \\
\hline Resistência a penetração média $(\mathrm{kPa})$ & $511,30^{\mathrm{b}}$ & $513,30^{\mathrm{b}}$ & $622,90^{\mathrm{a}}$ & $\mathrm{Kw} 8,23 \mathrm{e}^{-4^{*}}$ \\
\hline Resistência a penetração máxima (kPa) & $1.055,00^{\mathrm{b}}$ & $1.062,00^{\mathrm{b}}$ & $1.352,00^{\mathrm{a}}$ & $\mathrm{Kw} 9,44 \mathrm{e}^{-4^{*}}$ \\
\hline Resistência a penetração a 20 cm de profundidade $(\mathrm{kPa})$ & $944,70^{\mathrm{b}}$ & $902,90^{\mathrm{b}}$ & $1.238,00^{\mathrm{a}}$ & Av $9,44 \mathrm{e}^{-4^{*}}$ \\
\hline Cota $(m)$ & $1.627,00^{\mathrm{a}}$ & $1.338,00^{\mathrm{b}}$ & $1.656,00^{\mathrm{a}}$ & $\mathrm{Kw} 1,51 \mathrm{e}^{-10^{*}}$ \\
\hline Desnível máximo (m) & 3,90 & 2,87 & 3,41 & Av 0,34 \\
\hline Declividade média (graus) & 7,67 & 5,83 & 7,78 & Kw 0,19 \\
\hline
\end{tabular}

* Diferenças entre os setores significativas a 5\% de probabilidade, sendo as diferenças Av de acordo com a ANOVA (quando distribuição normal) e Kw de acordo com o Kruskal-Wallis (quando distribuição não normal). Médias seguidas pela mesma letra não diferem entre si estatisticamente pelos testes de Tukey (quando distribuição normal) e de comparação múltipla (quando distribuição não normal).

sobre os campos nativos no sul do Brasil; e Marchiori (2004) cita que em locais pedregosos as raízes pivotantes desenvolvem-se mais facilmente, possibilitando o estabelecimento de indivíduos arbóreos.

Outros elementos nucleadores encontrados foram árvores adultas vivas. $O$ fato dessas não terem apresentado relação com os regenerantes na Área 2 provavelmente foi devido à pouca abundância de árvores adultas na área (sete indivíduos). $\mathrm{O}$ sombreamento fornecido pelo crescimento de uma árvore (Padilla \& Pugnaire 2006) pode desfavorecer as gramíneas circundantes, o que possibilitaria o desenvolvimento de novas plântulas arborescentes. No entanto, devido a fase mais inicial de sucessão das áreas estudadas, poucos indivíduos adultos foram encontrados, não podendo ser comprovada sua atuação como elemento nucleador no presente caso.
Em todas as áreas, os elementos nucleadores não foram influentes sobre os indivíduos anemocóricos, sendo que outros fatores podem estar atuando no padrão espacial desses indivíduos. Conforme Negrini et al. (2012), outros fatores ecológicos, como a existência de filtros ambientais, podem atuar sobre o padrão de distribuição espacial dos propágulos. Esses fatores incluem a direção dos ventos, variáveis ambientais que estão atuando no estabelecimento dos regenerantes e a distância da fonte de propágulos.

Padrão semelhante de não relação com os elementos nucleadores foi encontrado para as autocóricas, encontradas somente na Área 2. Da mesma forma que para os indivíduos anemocóricos, os fatores do ambiente e os padrões de dispersão e de distância das fontes de propágulos provavelmente possuem maior influência na distribuição espacial desses indivíduos do que os elementos nucleadores. 
Entre as Áreas 1 e 2, apesar do histórico de perturbação e variáveis ambientes (a maioria) semelhantes, foram observados padrões de regeneração distintos, possivelmente influenciadas pelos diferentes elementos nucleadores encontrados nas áreas e pelas distintas cotas altitudinais. Mudanças de altitude podem influenciar, inclusive, na ocorrência de determinadas espécies, conforme já demonstrado em estudos de Higuchi et al. (2012) para a Floresta Ombrófila Mista. Assim, a presença abundante de xaxins mortos sobre o solo apenas na Área 1, que são resquícios do desmatamento pretérito, pode estar relacionada com a distribuição preferencial desta espécie ao longo de diferentes cotas altitudinais.

Observa-se que as Áreas 1 e 3 apresentam a mesma altitude, no entanto, seus históricos de perturbação distintos, aliado a características ambientais diferentes, provavelmente foram determinantes na baixa presença de regenerantes na Área 3, apesar de ambas apresentarem fonte de propágulos adjacentes. É difícil definir exatamente quais são os fatores que determinam a ocorrência de campo ou floresta nessas regiões de transição do Sul do Brasil. Porém, os resultados do presente estudo sugerem que as condições edáficas mais restritivas da Área 3, caracterizadas por um teor de areia superior a $80 \%$, elevado teor de alumínio e maior compactação do solo, supostamente provocada por pastoreio mais intensivo, possam estar dentre os fatores limitantes à regeneração de espécies lenhosas neste local. Assim, fica evidenciado que a limitação da regeneração do componente arbustivoarbóreo não seja o resultado de um único fator edáfico não favorável para as plantas, mas sim, pela combinação de condições restritivas.

Conclui-se que a distribuição espacial dos indivíduos pertencentes às guildas de dispersão e dos elementos nucleadores foi distinta entre as três áreas. Somente os indivíduos zoocóricos se associaram aos elementos nucleadores, indicando que estes funcionam como facilitadores para a dispersão pela fauna, não sendo os elementos relevantes para indivíduos dos outros tipos de dispersão encontrados.

Os elementos nucleadores mais efetivos foram os xaxins mortos, troncos e rochas, especialmente os dois primeiros, que foram os mais utilizados na área na qual os três elementos estavam disponíveis em elevada abundância (Área 1). As condições ambientais e históricos distintos entre as áreas imprimiram padrões de regeneração e relação com elementos nucleadores diferentes, porém, com convergências, especialmente em relação à importante relação da fauna com os elementos nucleadores, exceto na Área 3, distinta por ser área de campo no passado e, provavelmente, também devido às condições ambientais e ao pastoreio que pode ter reduzido a resiliência na área em proporcionar capacidade de regeneração de espécies arbustivo-arbóreas. Essas características distintas devem ser consideradas ao se analisar o avanço da floresta sobre o campo e na definição de técnicas de recuperação de áreas degradadas.

\section{Agradecimentos}

Ao CNPq (Edital Universal MCTI/CNPq 14/2014), o financiamento deste trabalho. À CAPES, a concessão de bolsa de Mestrado à primeira autora do trabalho. Ao CNPq, a concessão de bolsa de Produtividade para o segundo e o terceiro autores. Ao Parque Nacional de São Joaquim, em especial, ao M.Sc. Michel Tadeu Rodrigues Nolasco de Omena, a gentileza ao disponibilizar as áreas de estudo. À FAPESC, o apoio financeiro por meio do Edital PAP/UDESC. Ao Dr. Marcos Eduardo Guerra Sobral, a identificação de espécies de Myrtaceae. Ao Dr. Cláudio Augusto Mondin, a identificação de espécies de Asteraceae.

\section{Referências}

Almeida, S.R.; Watzlawick, L.F.; Myszka, E. \& Valerio, A.F. 2008. Florística e síndromes de dispersão de um remanescente de Floresta Ombrófila Mista em sistema faxinal. Ambiência 4: 289-297.

Aquino, C. \& Barbosa, L.M. 2009. Classes sucessionais e síndromes de dispersão de espécie arbóreas e arbustivas existentes em vegetação ciliar remanescente (Conchal, SP), como subsídio para avaliar o potencial do fragmento como fonte de propágulos para enriquecimento de áreas revegetadas no Rio Mogi-Guaçu, SP. Revista Árvore 33: 349-358.

Baddeley, A. \& Turner, R. 2005. Spatstat: an r package for analyzing spatial point patterns. Journal of Statistical Software 12: 1-42.

Bechara, F.C. 2006. Unidades demonstrativas de restauração ecológica através de técnicas nucleadoras: Floresta Estacional Semidecidual, Cerrado e Restinga. Tese de Doutorado. Universidade de São Paulo, Piracicaba, 249p.

Behling, H.; Pillar, V.P.; Orlóci, L. \& Bauermann, S.G. 2004. Late Quaternary Araucaria forest, grassland (Campos), fire and climate dynamics, studied by high-resolution pollen, charcoal and multivariate analysis of the Cambará do Sul core in southern Brazil. Palaeogeography, Palaeoclimatology, Palaeoecology 203: 277-297. 
Budke, J.C.; Athayde, E.A.; Giehl, E.L.H.; Záchia, R.A. \& Eisinger, S.M. 2005. Composição florística e estratégias de dispersão de espécies lenhosas em uma floresta ribeirinha, arroio Passo das Tropas, Santa Maria, RS, Brasil. Iheringia, Série Botânica 60: $17-24$.

Carlucci, M.B.; Duarte, L.S. \& Pillar, V.D. 2011. Nurse rocks influence forest expansion over native grassland in southern Brazil. Journal of Vegetation Science 22: 111-119.

Dale, M.R.T \& Fortin, M. 2014. Spatial analysis: a guide for ecologists. Cambridge University Press, Cambridge. $450 \mathrm{p}$.

Duarte, L.S.; Santos, M.M.G.; Hartz, S.M. \& Pillar, V.D. 2006. Role of nurse plants on Araucaria Forest expansion over grassland in South Brazil. Austral Ecology 31: 520-528.

Duarte, L.S.; Hofmann, G.S.; Santos, M.G.; Hartz, S.M. \& Pillar, V.D. 2010. Testing for the influence of niche and neutral factors on sapling community assembly beneath isolated woody plants in grassland. Journal of Vegetation Science 21: 462-471.

EMBRAPA. 2006. Sistema brasileiro de classificação de solos. $2^{\text {a }}$ ed. EMPRAPA-SPI, Rio de Janeiro. 306p.

Giraudoux, P. 2015. Pgirmess: data analysis in ecology. R package version 1.6.2, 2015. Disponível em $<$ http:// CRAN.R-project.org/package $=$ pgirmess $>$. Acesso em 18 março 2015.

Higuchi, P.; Silva, A.C.; Ferreira, T.S.; Souza, S.T.; Gomes, J.P.; Silva, K.M. \& Santos, K.F. 2012. Floristic composition and phytogeography of the tree component of Araucaria Forest fragments in southern Brazil. Brazilian Journal of Botany 35: 145-157.

Hijmans, R.J.; Cameron, S.E.; Parra, J.L.; Jones, P.G. \& Jarvis, A. 2005. Very high resolution interpolated climate surfaces for global land areas. International Journal of Climatology 25: 1965-1978.

IBGE. 2012. Manual técnico da vegetação brasileira. Fundação Instituto Brasileiro de Geografia e Estatística, Rio de Janeiro. 271p.

Klein, R. 1960. Aspectos dinâmico do pinheiro brasileiro. Sellowia 12: 17-44.

Marchiori, J.N.C. 2004. Fitogeografia do Rio Grande do Sul: Campos Sulinos. EST Edições, Porto Alegre. 15p.

Müller, S.C.; Overbeck, G.E.; Pfadenhauer, J. \& Pillar, V.P. 2012. Woody species patterns at forest- grassland boundaries in southern Brazil. Flora 207: 586-598.

Negrini, M.; Aguiar, M.D.; Vieira, C.T.; Silva, A.C. \& Higuchi, P. 2012. Dispersão, distribuição espacial e estratificação vertical da comunidade arbórea em um fragmento florestal no Planalto Catarinense. Revista Árvore 36: 919-930.

Oliveira Filho, A.T.; Vilela, E.A.; Carvalho, D.A. \& Gavilanes, M.L. 1994. Differenciation of streamside and upland vegetation in an area of montane semideciduous Forest in southeastern Brasil. Flora 189: 287-305.

Oliveira, J.M. \& Pillar, V.D. 2004. Vegetation dynamics on mosaics of Campos and Araucaria forest between 1974 and 1999 in Southern Brazil. Community Ecology 5: 197-202.

Padilla, F.M. \& Pugnaire, F.I. 2006. The role of nurse plants in the restoration of degraded environments. Frontiers in Ecology and the Environment 4: 196-202.

Pereira, I.M.; Botelho, S.A.; van den Berg, E.; Oliveira Filho, A.T. \& Machado, E.L.M. 2010. Caracterização ecológica de espécies arbóreas ocorrentes em ambientes de mata ciliar, como subsídio à recomposição de áreas alteradas nas cabeceiras do Rio Grande, Minas Gerais, Brasil. Ciência Florestal 20: 235-253.

Pillar, V.D. 2003. Dinâmica da expansão florestal em mosaicos de floresta e campos no Sul do Brasil. In: Claudino-Sales, V. (ed.). Ecossistemas brasileiros: manejo e conservação. Expressão Gráfica e Editora, Fortaleza. Pp. 209-216.

R Development Core Team. 2015. R: a language and environment for statistical computing. R Foundation for Statistical Computing. Disponível em <http:// www.R-project.org>. Acesso em 18 março 2015.

van der Pijl, L. 1982. Principles of dispersal in higher plants. Springer-Verlag, Berlim. 162p.

Wiegand, T.; Moloney, K.A.; Naves, J. \& Knauer, F. 1999. Finding the missing link between landscape structure and population dynamics: a spatially explicit perspective. The American Naturalist 154: 605-627.

Wiegand, T. \& Moloney, K.A. 2004. Rings, circles, and null-models for point pattern analysis in ecology. Oikos 104: 209-229.

Yarranton, G.A. \& Morrison, R.G. 1974. Spatial dynamics of a primarysuccession: nuclation. Jornal of Ecology 62: 417-428. 